\title{
Collective polarization effects in $\beta$-polyvinylidene fluoride and its copolymers with tri- and tetrafluoroethylene
}

\author{
S. M. Nakhmanson, ${ }^{1}$ M. Buongiorno Nardelli, ${ }^{2,3}$ and J. Bernholc ${ }^{2,3}$ \\ ${ }^{1}$ Department of Physics and Astronomy, Rutgers University, Piscataway, New Jersey 08854, USA \\ ${ }^{2}$ Center for High Performance Simulation and Department of Physics, North Carolina State University, \\ Raleigh, North Carolina 27695, USA \\ ${ }^{3}$ Center for Computational Sciences (CCS) and Computer Science and Mathematics Division, \\ Oak Ridge National Laboratory, Oak Ridge, Tennessee 37830, USA \\ (Received 22 May 2005; revised manuscript received 8 August 2005; published 23 September 2005)
}

\begin{abstract}
The polar properties of the $\beta$ phase of polyvinylidene fluoride (PVDF) and its copolymers with tri- and tetrafluoroethylene are investigated theoretically at different VDF-to-copolymer ratios. The calculations show that polarization in such polymers is described by cooperative, quantum-mechanical interactions between polymer chains, which cannot be viewed as a superposition of rigid dipoles. For $\beta$-PVDF, the monomer dipole moment is increased by $50 \%$ (from 2 to $3 \mathrm{D}$ ) as the isolated chains are brought together to form a crystal. In PVDF crystals containing copolymers, we observe a weakly parabolic dependence of monomer dipole moments on copolymer concentration.
\end{abstract}

DOI: 10.1103/PhysRevB.72.115210

PACS number(s): 61.41.+e, 77.70.+a

\section{INTRODUCTION}

Ferroelectric materials are critically important for a wide range of current and prospective technological applications. These are not limited to various electromechanical devices, like sensors, actuators, and transducers, but also include nonvolatile ferroelectric memories, high- $k$ dielectrics for modern microelectronics, pyroelectric arrays, and acoustic and nonlinear optical devices. Unfortunately, however, there exist only very few classes of materials that exhibit ferroelectric behavior. Perovskite-type $\mathrm{ABO}_{3}$ metal oxides (e.g., $\mathrm{BaTiO}_{3}$ or $\mathrm{KNbO}_{3}$ ), comprising the most important class of ferroelectric materials, are widely known for their strong polarization and excellent piezoelectric response. Still, their usefulness is limited by heavy weight, brittleness, and substantial costs of device manufacturing. Furthermore, the highest polarization and piezolectricity are achieved in perovskites containing lead (e.g., $\mathrm{PbZr}_{1-x} \mathrm{Ti}_{x} \mathrm{O}_{3}$ ), while the properties of lead-free compounds, especially when grown as thin films, are more modest. ${ }^{1}$ Ferroelectric polymers, on the other hand, exhibit properties almost complementary to those of perovskite-type oxides. In addition to being respectable piezo- and pyroelectrics, they are light, flexible and nontoxic, ${ }^{2-7}$ which is particularly important in the view of the recent interest in new, more efficient, and environmentally friendly materials.

Polyvinylidene fluoride (PVDF), $\left[-\mathrm{CH}_{2}-\mathrm{CF}_{2}-\right]_{n}$, the most well-known ferroelectric polymer, is a complex material that is usually synthesized as a mixture of ordered and disordered phases, and requires some postprocessing in the form of stretching and poling to become ferroelectric (see, e.g., Figs. $3-5$ in Ref. 2 for a good illustration). The most polar all-trans $\beta$ phase of PVDF, shown in Fig. 1, has been extensively studied by theorists during the past three decades, which resulted in a number of empirical models for polarization in $\beta$-PVDF. ${ }^{8-14}$ Since, even after the aforementioned postprocessing procedure, $\beta$-PVDF is usually only
$50 \%$ crystalline, ${ }^{15}$ until very recently there had been no certainty about the value of polarization in the fully crystalline material. As can be seen from Table I, the various models do not agree with each other, which recently motivated us to apply modern $a b$ initio techniques to compute spontaneous polarization in $\beta$-PVDF. ${ }^{16}$ Our results $\left(P_{3}^{\mathrm{sp}}=0.178 \mathrm{C} / \mathrm{m}^{2}\right)$ clearly show that the majority of the previous models, fitted either to experiment or to a simple sum of the bond-dipole moments in an individual VDF monomer ${ }^{4,7}(\simeq 2 \mathrm{D})$, underestimate the strength of the dipole-dipole interaction in this material. The polarization obtained in Ref. 13, utilizing a force field developed by fitting to Hartree-Fock calculations for finite PVDF chains, ${ }^{17}$ is the only notable exception,

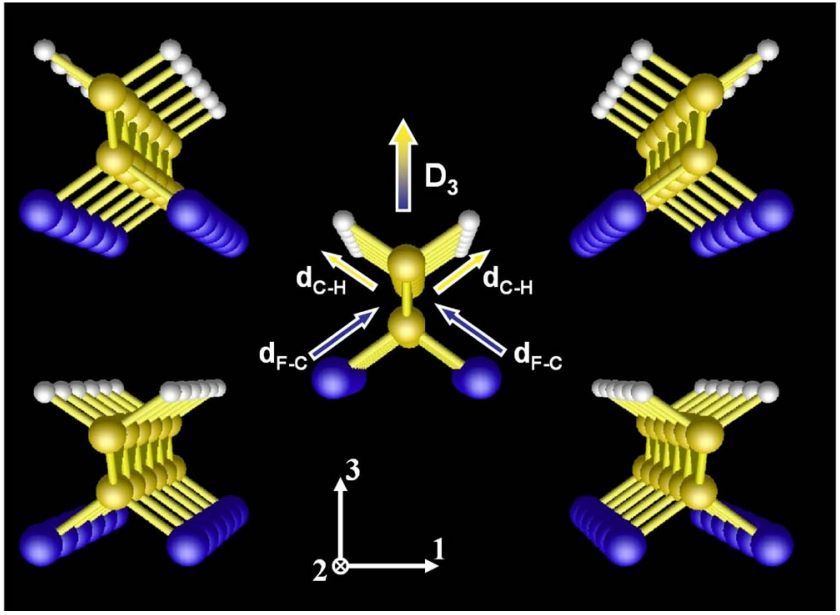

FIG. 1. (Color online) The structure of the crystalline $\beta$ phase of PVDF. The polar-covalent nature of the $\mathrm{F}-\mathrm{C}$ and $\mathrm{C}-\mathrm{H}$ bonds leads to polarization in the VDF monomer, directed perpendicular to the carbon backbone; $\boldsymbol{d}_{\mathrm{F}-\mathrm{C}}$ and $\boldsymbol{d}_{\mathrm{C}-\mathrm{H}}$ are the bond-dipole moments. The unit cell coordinate system is shown at the bottom with " 2 " and " 3 " being the backbone and polarization directions, respectively. 
TABLE I. Polarization $P^{\mathrm{sp}}$ in $\beta$-PVDF computed with various theoretical models. $C$ is the crystallinity of the material.

\begin{tabular}{lcccc}
\hline \hline Model & Year & $C(\%)$ & $P_{3}^{\mathrm{sp}}\left(\mathrm{C} / \mathrm{m}^{2}\right)$ & Ref. \\
\hline Rigid dipoles & & 100 & 0.131 & 4 \\
Kakutani, Mopsik and Broadhurst & $1970-75$ & 100 & 0.22 & 8 and 9 \\
Tashiro et al. & 1980 & 100 & 0.140 & 10 \\
Purvis and Taylor & $1982-83$ & 100 & 0.086 & 11 \\
Al-Jishi and Taylor & 1985 & 100 & 0.127 & 12 \\
Carbeck et al. & 1995 & 100 & 0.182 & 13 and 14 \\
Nakhmanson et al. & 2004 & 100 & 0.178 & 16 \\
Experiment & & $\approx 50$ & $0.05-0.08$ & \\
\hline \hline
\end{tabular}

stressing the idea that "ab initio input" is required to produce an accurate model. However, neither of the previous investigations studied the electrostatic interactions among monomers in $\beta$-PVDF-type polymer structures at the detailed level required to fully elucidate the intricacies of their polar behavior.

Moreover, although the ideal $\beta$-PVDF structure serves well as a simple model for basic understanding of the polar nature of ferroelectric polymer crystals, it is quite different from the polymeric structures used in actual device manufacturing. Since poor crystallinity of "pure" PVDF degrades its polar properties, it is usually mixed with trifluoroethylene (TrFE), [- $\left.\mathrm{CHF}-\mathrm{CF}_{2}-\right]$, and tetrafluoroethylene (TeFE), $\left[-\mathrm{CF}_{2}-\mathrm{CF}_{2}-\right]$ (shown in Fig. 2) to produce device-grade materials. Naturally, compared to VDF, TrFE and TeFE monomers exhibit lower or no polarization. However, such $\mathrm{P}(\mathrm{VDF}-$-copolymer) systems can be grown with $90 \%$ or better crystallinity, ${ }^{3,18-21}$ which results in stronger polarization and piezoelectric response than that of $50 \%$ crystalline PVDF. In addition, these structures can be grown in the form of ultrathin films ${ }^{22}$ and have other important electromechanical properties, e.g., giant electrostriction. ${ }^{23}$ However, it turns

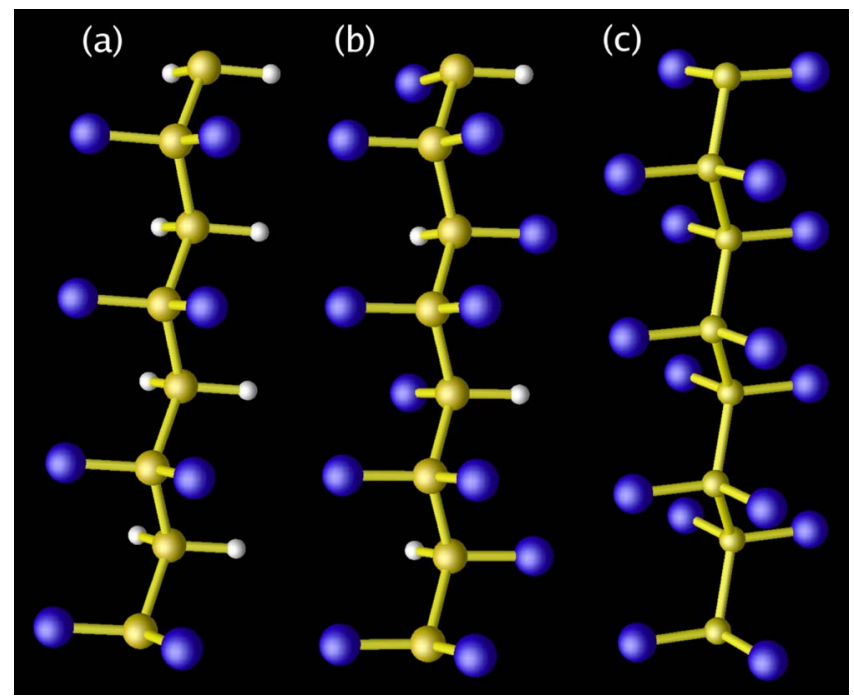

FIG. 2. (Color online) Chain models for PVDF and copolymers. (a) PVDF chain in all-trans conformation. (b) Poly-TrFE chain with alternating hydrogens. (c) Poly-TeFE chain. out that realistic $a b$ initio models of polarization in these materials are required to explain the peculiarities of their behavior and to open avenues for development of better polymeric ferroelectrics for modern technological applications.

In order to fully understand the physics of polarization in crystalline $\beta$-PVDF and in $\mathrm{P}(\mathrm{VDF}$-copolymer) systems one needs to answer the following questions. (a) What are the main "building blocks" governing the polar properties of a polymer-ferroelectric crystal: are these individual monomers, chains, or, possibly, something else? (b) What is the "natural value" of a structural unit dipole moment in a given environment (individual molecule, chain, crystal)? (c) What happens to this value when the environment changes, e.g., when the VDF-to-copolymer concentration changes?

The rest of this paper is organized as follows. Section II briefly reviews the formulation of modern polarization theory in terms of Wannier functions and presents the details of the numerical techniques that were used to compute polarization. In Sec. III we discuss the results of our calculations, progressing from individual monomers to chains and then to P(VDF-copolymer) crystals. Finally, Sec. IV presents the summary and the conclusions.

\section{METHODOLOGY}

\section{A. Modern theory of polarization}

In an infinite periodic solid, the macroscopic polarization can be written as the sum of ionic and electronic terms:

$$
\boldsymbol{P}=\boldsymbol{P}_{\text {ion }}+\boldsymbol{P}_{\mathrm{el}}=\frac{e}{V} \sum_{\tau} Z_{\tau} \boldsymbol{b}_{\tau}-\frac{1}{V} \int_{V} \boldsymbol{r} \rho(\boldsymbol{r}) d \boldsymbol{r},
$$

where $V$ is the volume of the unit cell, $Z_{\tau}$ and $\boldsymbol{b}_{\tau}$ are the charge and position of the $\tau$ th atom in the cell, and $\rho(\boldsymbol{r})$ is the cell-periodic density of electron charge. Since the position operator $\boldsymbol{r}$ lacks the periodicity of the system and $\rho(\boldsymbol{r})$ is usually delocalized in covalent or polar-covalent structures, the second term of the equation has to be regularized before further use. The de facto standard approach for such a regularization was proposed by the modern theory of polarization, put forward about a decade ago. ${ }^{24}$ The theory provides two complementary techniques to obtain $\boldsymbol{P}_{\mathrm{el}}$, depending on the particular representation for $\rho(\boldsymbol{r})$, which can be expanded 


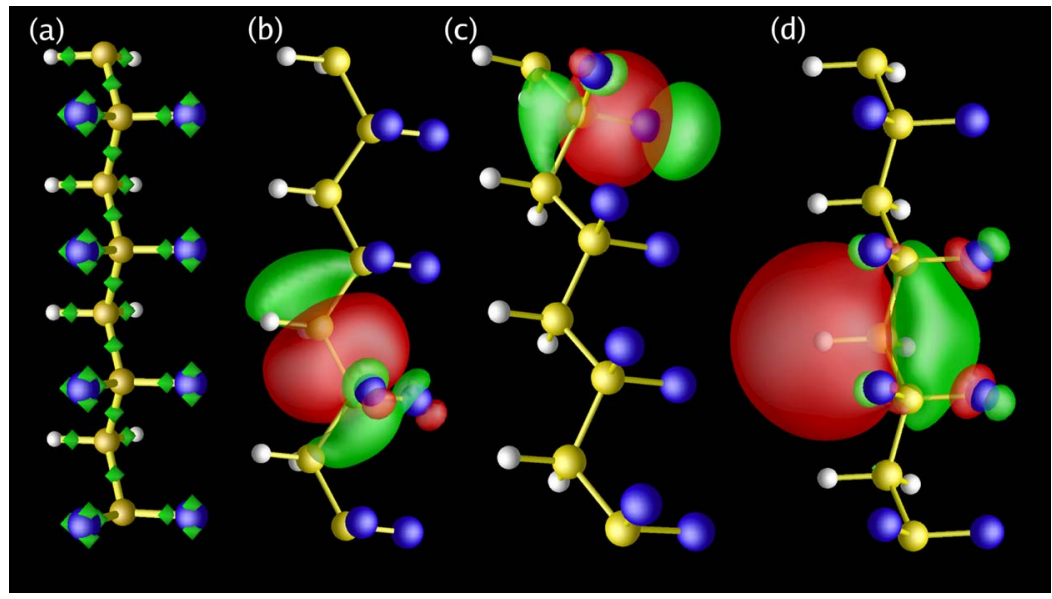

FIG. 3. (Color online) MLWFs and their charge centers computed for an isolated infinite $\beta$-PVDF chain. (a) Diamonds mark the positions of the MLWF charge centers on the chain. (b)-(d) Typical MLWFs localized on carbon-carbon, carbon-fluorine, and carbon-hydrogen bonds, respectively. into either a sum of occupied Bloch states $\psi_{i k}(\boldsymbol{r})$ or a sum over occupied Wannier functions (WFs) $W_{i}(\boldsymbol{r})$ (for pairedelectron systems):

$$
\rho(\boldsymbol{r})=-\frac{2 e}{(2 \pi)^{3}} \sum_{i \text { occ }} \int_{B Z} d \boldsymbol{k}\left|\psi_{i k}(\boldsymbol{r})\right|^{2}=-2 e \sum_{i \text { occ }}\left|W_{i}(\boldsymbol{r})\right|^{2} .
$$

Utilizing the Bloch-state representation for $\rho(\boldsymbol{r})$ leads to a widely known "Berry-phase" expression for electronic polarization, which works well when only the total value of $\boldsymbol{P}_{\mathrm{el}}$ is needed. If, however, one is interested in contributions to polarization originating from various structural units in the system, e.g., individual dipole moments of monomers in a polymer crystal, or "layer-by-layer" polarizations in a perovskite ferroelectric, these values cannot be extracted from a single Berry-phase computation. ${ }^{25}$

On the other hand, the WF representation for $\rho(\boldsymbol{r})$ produces an especially transparent formula for $\boldsymbol{P}_{\mathrm{el}}$ useful for polarization analysis in "modular" systems: ${ }^{26}$

$$
\boldsymbol{P}_{\mathrm{el}}=-\frac{2 e}{V} \sum_{i \text { occ }}\left\langle W_{i}|\boldsymbol{r}| W_{i}\right\rangle=-\frac{2 e}{V} \sum_{i \text { occ }}\left\langle\boldsymbol{r}_{i}\right\rangle,
$$

where $\left\langle\boldsymbol{r}_{i}\right\rangle$ are the centers of charge of WFs $W_{i}(\boldsymbol{r})$. Compared to Eq. (1), $\boldsymbol{P}_{\mathrm{el}}$ in the equation above is well defined for sufficiently localized $W_{i}(\boldsymbol{r})$. A general algorithm, outlined in Refs. 27 and 28, exploits the nonuniqueness of the unitary transformation connecting Bloch orbitals $\psi_{i k}(\boldsymbol{r})$ and WFs $W_{i}(\boldsymbol{r})$ to iteratively find maximally localized Wannier functions (MLWFs). The sum of quadratic spreads of the Wannier probability distributions $\left|W_{i}(\boldsymbol{r})\right|^{2}$,

$$
\Omega=\sum_{i \text { occ }}\left[\left\langle r_{i}^{2}\right\rangle-\left\langle\boldsymbol{r}_{i}\right\rangle^{2}\right]
$$

is used as a minimization criterion. In general, this transformation has a $k$-space dependence, which makes calculations more complicated. However, for the studies of polymer chains and crystals we can use large supercells, in which the $\Gamma$-point approximation for the $k$-space summation is well justified. In this particular case, computing MLWFs is reduced to finding a single orthonormal transformation matrix, which can be done very efficiently by a number of numerical techniques. ${ }^{29,30}$

As already pointed out above, expression (3) is particularly useful for studies of "modular" systems, like polymer chains or crystals. Because in such systems each structural unit (monomer) is connected to only a single neighbor from above and below, one can easily identify the WF centers "belonging" to the unit as well as the ones that are shared with the neighbors. Combining Eqs. (1) and (3), a simple formula for the structural unit's dipole moment can be obtained:

$$
\boldsymbol{D}=e \sum_{\tau \in \text { unit }} Z_{\tau} \boldsymbol{b}_{\tau}-2 e \sum_{i \in \text { unit }}\left\langle\boldsymbol{r}_{i}\right\rangle
$$

Here we implicitly assume that contributions from the shared centers are included with weights of one-half. This expression will be extensively used for the studies of the monomers' dipole moments, presented in the next section.

\section{B. Computational details}

For all the calculations presented here, we used an $a b$ initio multigrid-based total-energy method, which employs a real-space grid as a basis ${ }^{31}$ and three-dimensional periodic boundary conditions. The exchange-correlation interaction was described by the generalized gradient approximation of Ref. 32. We employed norm-conserving pseudopotentials ${ }^{33,34}$ generated by the FHI98PP package. ${ }^{35}$ The $\Gamma$-point-specific routine for calculating the MLWFs was developed using the simultaneous diagonalization algorithm of Ref. 30. This algorithm allows for bypassing the computation of the WFs if only their charge centers are needed.

Although van der Waals interactions are neglected in density functional theory (DFT), the cohesion in highly polar structures, such as $\beta$-PVDF, is dominated by dipole-dipole interactions, which are well described by DFT.

For cell-volume relaxations we used a plane-wave-based PWSCF code $^{36}$ and ultrasoft pseudopotentials, ${ }^{37}$, to simultaneously relax the cells and ionic positions in the P(VDFcopolymer) systems. The optimized cells were then used in WF calculations with the grid-based method. ${ }^{38}$

An example result of a MLWF calculation for an isolated infinite $\beta$-PVDF chain is presented in Fig. 3. The leftmost 
TABLE II. Average monomer dipole moments in the infinite PVDF, PTrFE, and PTeFE chains. Values computed at $\beta$-PVDF chain-backbone lattice constant $(2.56 \AA$ / unit $)$ as well as at the relaxed lattice constant are shown.

\begin{tabular}{lccc}
\hline \hline & $\begin{array}{c}D_{3}, c=2.56 \AA \text { /unit } \\
(\mathrm{D})\end{array}$ & $\begin{array}{c}D_{3} \text {, relaxed } c \\
(\mathrm{D})\end{array}$ & $\begin{array}{c}\text { Relaxed } c \\
(\AA / \text { unit })\end{array}$ \\
\hline $\mathrm{VDF}$ & 2.00 & 2.00 & 2.560 \\
$\mathrm{TrFE}$ & 0.78 & 0.75 & 2.667 \\
$\mathrm{TeFE}$ & Nonpolar & Nonpolar & 2.749 \\
\hline \hline
\end{tabular}

panel (a) shows MLWF charge centers on a chain, with a single center located on every $\sigma$ bond in the system and three additional centers localized near each fluorine atom. The remaining three panels show MLWFs localized on different types of $\sigma$ bonds: (b) carbon-carbon, (c) carbon-fluorine, and (d) carbon-hydrogen.

\section{RESULTS AND DISCUSSION}

\section{A. From a monomer to a chain}

The first question to be addressed is what happens to the value of the monomer's dipole moment $\boldsymbol{D}$ given by Eq. (5), as an increasing number of monomers is being united into a chain.

In order to answer this question, we computed average dipole moments of VDF, TrFE, and TeFE structural units in finite $\mathrm{H}-\left[\mathrm{CH}_{2}-\mathrm{CF}_{2}\right]_{n}-\mathrm{H}, \mathrm{H}-\left[\mathrm{CHF}-\mathrm{CF}_{2}\right]_{n}-\mathrm{H}$, and $\mathrm{H}-\left[\mathrm{CF}_{2}-\mathrm{CF}_{2}\right]_{n}-\mathrm{H}$ polymer chains for $n=1, \ldots, 8$. The chains were relaxed to ionic forces of less than $0.025 \mathrm{eV} / \AA$ in large supercells, so that the distance between each chain and its periodic images was approximately $10 \AA$. In addition, we computed the same dipole moments for the limiting case of infinite chains, like the ones shown in Fig. 2, in supercells containing four or eight structural units. The calculations were performed at both $\beta$-PVDF ( $2.56 \AA$ per structural unit) and the fully relaxed lattice constants $c$ along the chainbackbone direction. The results are presented in Table II. Our value of the VDF dipole moment is in excellent agreement with results in the literature. ${ }^{7,39}$

Figure 4 shows the average values of VDF, TrFE, and TeFE monomer dipole moments in finite hydrogenterminated chains. Since the terminating hydrogens alter the values of the dipole moments of the first and the last units in the chain, we included two sets of curves for each monomer type, with the chain-end units included and excluded from the averaging process. In both cases the curves for the monomer dipoles in finite chains quickly converge to their infinite, relaxed $c$, chain values on the right border of the figure. In general, we observe that apart from very short chains ( $n$ $=1-3$ ), the average value of the monomer dipole moment is very close to its infinite-chain limit. This shows that in a single chain there is no polarization enhancement due to the growing electric field produced by the neighboring units.

Similar results are obtained for polymer chains where one or more monomers are substituted by copolymers. The results of such calculations for the chains where one of the

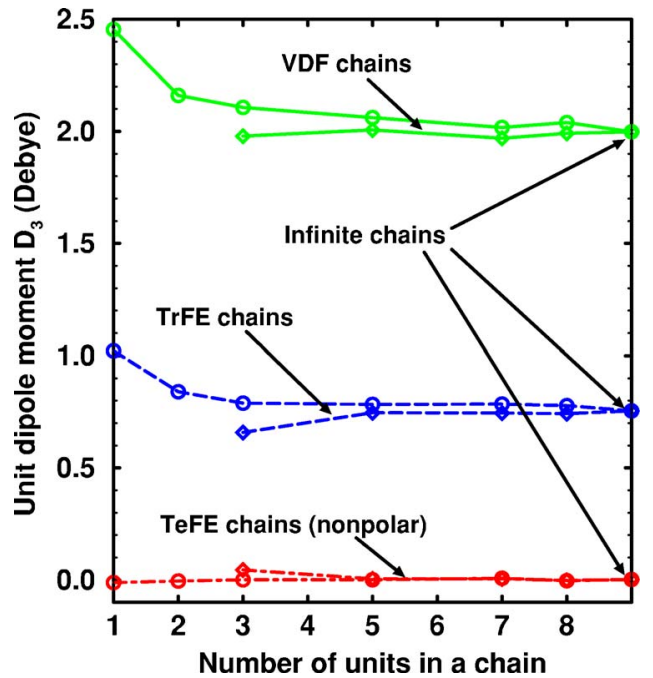

FIG. 4. (Color online) Variation of the dipole moment $D_{3}$ in VDF, TrFE, and TeFE monomer units in finite chains with increasing numbers of units. Dipole moments obtained by averaging over all $n$ monomers in a chain are shown as circles, while diamonds represent the average moments when the end units (i.e., those in direct contact with terminating hydrogens) are excluded. The dipole moments of the monomers in infinite chains are shown on the right.

eight units in the supercell was substituted by TrFE, TeFE, or an inverted VDF, also known as the head-to-head, tail-to-tail (HHTT) defect, are presented in Fig. 5. All of these cases demonstrate that introduction of an isolated defect, even as drastic as HHTT, into a $\beta$-PVDF chain does not seriously affect the polar states of the other units in the chain, except maybe the nearest neighbors of the substituted unit. On the other hand, a copolymer unit or a HHTT defect substituted into the chain keeps its own characteristic features fairly intact or even amplifies them. For example, a TrFE unit embedded into the chain has the same $D_{3}$ component as in a PTrFE chain and it also retains its large $D_{1}$ component. Similarly, the HHTT defect has its $D_{3}$ component increased to nearly $3 \mathrm{D}$.

We can conclude that (i) in general, individual monomers in infinite isolated chains prefer to "keep their identities," and (ii) various kinds of elaborate polarization patterns in a chain can be created by assembling the appropriate monomer types. In this respect a $\left(-\left[\mathrm{CH}_{2}-\mathrm{CF}_{2}\right]-[\mathrm{CHF}-\mathrm{CHF}]-\right)_{n}$ system will be particularly interesting, with VDF units having large $D_{3}$ and [- $\mathrm{CHF}-\mathrm{CHF}-]$ units large $D_{1}$ projections. Of course, such level of precise monomer manipulation has not yet been achieved, but it may become possible in the future.

\section{B. From a chain to a crystal}

After understanding the behavior of monomer structural units in isolated chains, we investigate the "structural assembly" of chains. Starting from an isolated infinite chain, we gradually bring the chains together until a crystal is formed.

We computed the average VDF dipole moments for a system containing four $\beta$-PVDF chain segments of four units each in a pseudohexagonal arrangement in an orthorhombic 


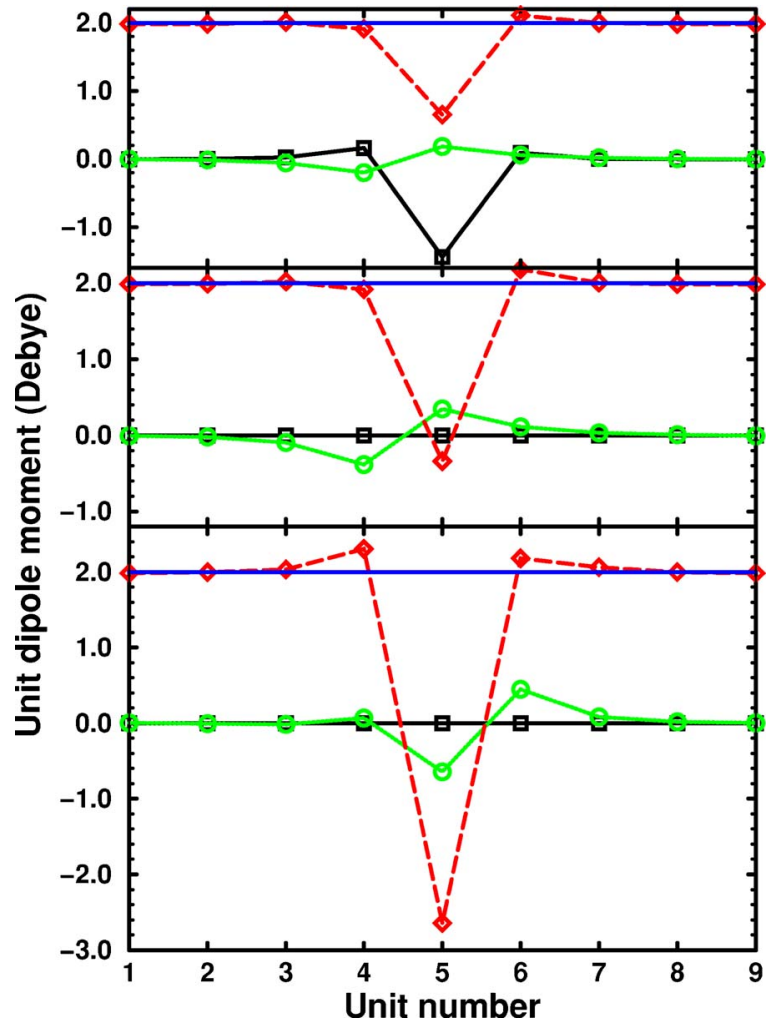

FIG. 5. (Color online) Structural unit dipole moment components $D_{1}$ (squares), $D_{2}$ (circles), and $D_{3}$ (diamonds) in infinite isolated $\beta$-PVDF chains where one of the units (number 5 in this figure) was substituted by TrFE (top panel), TeFE (middle panel), and inverted VDF (bottom panel). Horizontal lines at $2 \mathrm{D}$ in each panel mark the $D_{3}$ value of the VDF unit in a $\beta$-PVDF chain with no substitutions.

supercell. The lattice constant $c$ in the backbone direction was fixed at $2.56 \AA$ / unit, while the lattice constants $a$ and $b$ were allowed to vary. Two calculations were carried out for each particular interchain distance (i.e., a combination of $a$ and $b$ ). In the unrelaxed case, the ions were held fixed (in the same arrangement as for the relaxed isolated chain), so that only the electronic degrees of freedom were allowed to vary. In the relaxed-chain case, both the electrons and the ions were allowed to relax to their minimum-energy positions. The ionic forces in the relaxed configuration were smaller than $0.025 \mathrm{eV} / \AA$. The polarization of the crystalline structure was computed at the experimental lattice constants of $a=8.58 \AA, \quad b=4.91 \AA$ (polarization direction), and $c$ $=2.56 \AA$ (backbone direction) for the primitive orthorhombic cell with two structural units. The actual supercell used in the calculations for the crystal contained $1 \times 2 \times 4$ primitive cells.

The results of the calculations are presented in Fig. 6, where the average VDF dipole moments are plotted vs the interchain distance. The most striking feature of these curves is a dramatic increase in the VDF dipole moment as the chains are brought out from the noninteracting limit and assembled into the crystalline $\beta$-PVDF structure. A similar comparison was also performed in Ref. 13, using the $a b$ initio-fitted force field. ${ }^{17}$ Although the values of VDF unit

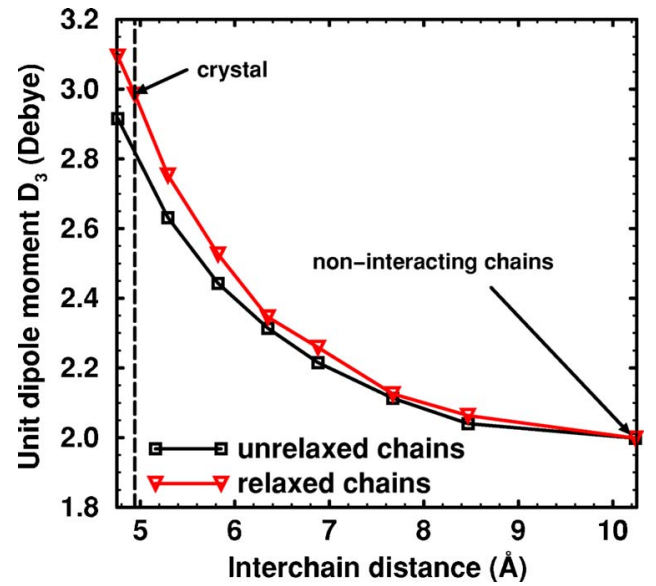

FIG. 6. (Color online) Average $D_{3}$ component of the VDF unit dipole moment in an infinite $\beta$-PVDF chain as the chains are brought together to form a crystal. See text.

dipole moments in these calculations do not perfectly agree with our results, the degree of the polarization enhancement between the isolated chain and the crystal is nearly the same as the one obtained in this work.

Apart from the fact that the "collective polarization" enhancement in the $\beta$-PVDF crystal is much stronger than that in the isolated chain, the polarization curves of Fig. 6 show why many of the other empirical models in Table I give polarization in $\beta$-PVDF that is too low. Apparently, using the value of the VDF dipole moment of an isolated chain in a crystal (which is tempting since the former is well known) completely neglects the "collective polarization" enhancement effect. $A b$ initio calculations unequivocally show that this effect is very large and has to be accounted for in order to get an accurate value for polarization in a fully crystalline $\beta$-PVDF. However, in addition to simply computing the magnitude of the polarization enhancement, our approach allows for an effective decoupling of the electronic and ionic contributions to it. If we compare the curves for the unrelaxed and relaxed chains in Fig. 6, we notice that even in the former case, i.e., with ions pinned in place, we still obtain $80 \%$ of the total polarization enhancement effect, indicating that the nature of the polarization increase is mostly electronic. Indeed, the calculations show that ionic relaxations only lead to an aproximately $1^{\circ}$ narrowing of the $\mathrm{F}-\mathrm{C}-\mathrm{F}$ angle in a VDF monomer, leaving all of the bond lengths practically unchanged. On the other hand, the centers of WFs that are attached to a monomer's $\mathrm{C}-\mathrm{F}$ and $\mathrm{C}-\mathrm{H}$ bonds shift by $1-3 \%$. The magnitudes of these shifts are on the order of $0.1 \mathrm{bohr}$, which is very small, but sufficient to produce the observed dipole-moment change of about $1 \mathrm{D}$.

\section{Crystal with copolymer units}

Starting from the $\beta$-PVDF crystal we substituted some of the VDF units by TrFE and TeFE to create realistic models of $\mathrm{P}$ (VDF-copolymer) crystalline systems and to study their structural and polar properties. Since TrFE and TeFE structural units contain more "bulky" fluorine atoms than VDF, 


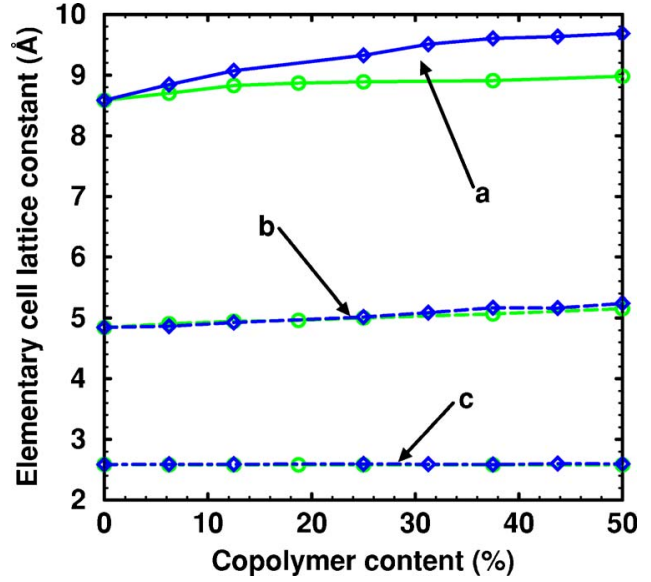

FIG. 7. (Color online) Evolution of an elementary two-unit orthorhombic cell lattice constants with changing copolymer concentration. Circles and diamonds represent the $\mathrm{P}(\mathrm{VDF}-\mathrm{TrFE})$ and $\mathrm{P}(\mathrm{VDF}-\mathrm{TeFE})$ families, respectively.

simultaneous relaxations of the cells and ionic positions in the $\mathrm{P}(\mathrm{VDF}$-copolymer) systems are required. In these calculations, one to eight units in the initial $\beta$-PVDF crystal were changed into TrFE or TeFE, which corresponds to copolymer concentrations of 6.25-50\%. Each P(VDF-copolymer) structure was relaxed to stresses of less than $0.5 \mathrm{kbar}$ and ionic forces of less than $0.025 \mathrm{eV} / \AA$.

In Fig. 7 we show the changes in the lattice constants (reduced to the primitive $\beta$-PVDF orthorhombic cell with two units) as VDF units in the system are being turned into either TrFE or TeFE. Clearly, there is no expansion along the direction of the carbon backbone-the only "rigid" direction in the system-and only a weak expansion in the polarization direction in both families of models. This leaves the bulk of volume expansion to occur through the increasing lattice constant $a$. For the 50-50 VDF-to-copolymer concentration, in $\mathrm{P}(\mathrm{VDF}-\mathrm{TrFE})$ and $\mathrm{P}(\mathrm{VDF}-\mathrm{TeFE})$ systems $a$ grows by $4.6 \%$ and $12.8 \%$, respectively. In Fig. 8 we plot the evolution of an average dihedral angle between the neighboring units in the system with growing copolymer concentration. For $\beta$-PVDF our calculations produce dihedral angles that are very close to $180^{\circ}$, which means that neighboring units on a chain are all perfectly aligned in the polar direction and agrees with the previous first-principles calculations of Ref. 17. This result does not support the model of Hasegawa et al. ${ }^{40}$ who suggest that in $\beta$-PVDF monomers in a chain are tilted by $7^{\circ}$ with respect to each other due to the steric hindrance of the neighboring fluorine atoms. (The tilt angle is defined as $180^{\circ}$ minus the dihedral angle.) However, our calculations show that $\beta$-PVDF does develop tilts when some units are changed into TrFE or TeFE. In fact, the presence of only a few TrFE monomers (one or two units in the 16 unit cell, equivalent to the defect concentration of $5-10 \%$ ), which have nonzero dipole moment components along the " 3 " and " 1 " directions, produces a tilt of around $7^{\circ}$. On the other hand, dihedral tilts develop more gradually for the TeFE monomers that do not possess nonzero dipole moment components along the "1" direction. At 50-50 VDF-tocopolymer ratio, both $\mathrm{P}(\mathrm{VDF}-\mathrm{TrFE})$ and $\mathrm{P}(\mathrm{VDF}-\mathrm{TeFE})$ have

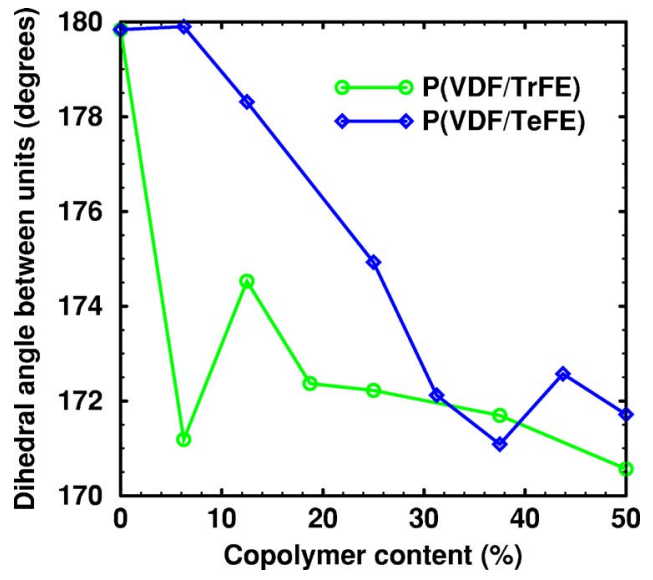

FIG. 8. (Color online) Evolution of the average dihedral angle between monomers in the $\mathrm{P}(\mathrm{VDF}-\mathrm{TrFE})$ (circles) and $\mathrm{P}(\mathrm{VDF}-$ $\mathrm{TeFE}$ ) (diamonds) families with changing copolymer concentration.

dihedral angles of $170^{\circ}-172^{\circ}$ indicating a less compact monomer arrangement in a chain, i.e., the fluorine atoms "stick out" more. This occurs mostly along the " 1 " direction, resulting in an increase in $a$ lattice constant.

The observed sensitivity of the dihedral angles in P(VDFcopolymer) mixtures to the presence of asymmetric defects may remove an inconsistency in the conclusions of Hasegawa et al., who obtained better agreement between the measured and the computed structure factors by introducing alternating monomer tilts into their model, but saw no evidence of the appropriate cell-doubling along the backbone direction in their x-ray patterns. It is therefore possible that the actual monomer tilts in their samples were due to the presence of various defects, which would explain the improvement in the structure factor after introducing the tilt. At the same time, the random distribution of defects in a sample explains the absence of the cell-doubling line in the x-ray spectra. In any case, it would be very interesting to refine this experiment using $\beta$-PVDF samples produced by modern growth techniques that allow for better control of defect formation and crystallinity.

Turning to the polar properties of $\mathrm{P}(\mathrm{VDF}$-copolymer) crystalline models, we computed copolymer concentrationrelated changes in the dipole moment of every type of monomer (VDF, TrFE, or TeFE) in each model family. The results are shown in Fig. 9, with the limiting values for monomer dipole moments in isolated infinite chains included for comparison. Clearly, the VDF dipole moment decreases in both $\mathrm{P}(\mathrm{VDF}-\mathrm{TrFE})$ and $\mathrm{P}(\mathrm{VDF}-\mathrm{TeFE})$ with growing copolymer concentration. However, even at 50-50 VDF-to-copolymer ratio, the VDF unit dipole moments are still approximately $20 \%$ larger than those in an isolated $\beta$-PVDF chain. The TrFE and TeFE monomer dipole moments in P(VDFcopolymer) crystals demonstrate a strongly nonlinear behavior with respect to copolymer concentration. At TrFE concentration of less than $20 \%$, the TrFE unit in a crystal is more than twice as polar as in an isolated chain. At 50-50 VDFto-TrFE concentration, it is still $40 \%$ more polar than that of an isolated chain. Analogously, in a crystal with TeFE concentration of less than $20 \%$, this naturally nonpolar monomer 


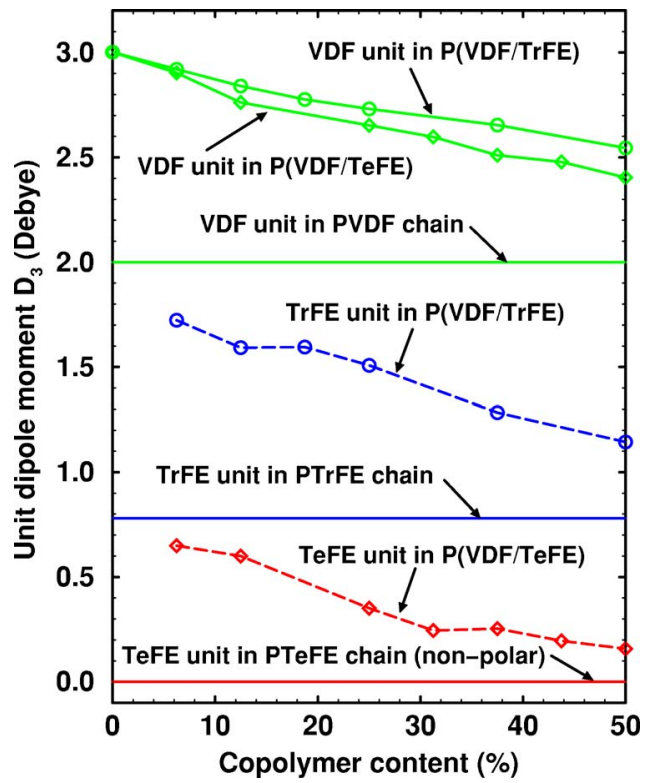

FIG. 9. (Color online) Changes in the values of average dipole moments of VDF, TrFE, and TeFE structural units in the crystalline $\mathrm{P}(\mathrm{VDF}-\mathrm{TrFE})$ and $\mathrm{P}(\mathrm{VDF}-\mathrm{TeFE})$ models with increasing copolymer concentration. The limiting values of unit dipole moments in the isolated infinite $\beta$-PVDF, PTrFE, and PTeFE chains are shown for comparison.

has a dipole moment of $0.6-0.7 \mathrm{D}$ and it stays polar even at 50-50 VDF-to-TeFE concentration.

Finally, in Fig. 10 we present the copolymer concentration dependence for the total polarization of the crystalline $\mathrm{P}(\mathrm{VDF}-\mathrm{TrFE})$ and $\mathrm{P}(\mathrm{VDF}-\mathrm{TeFE})$ models. The curves for both families closely follow the behavior of VDF dipole moments in Fig. 9, displaying a similar downward bowing, which is a bit more pronounced in the case of $\mathrm{P}(\mathrm{VDF}-\mathrm{TeFE})$. A fit to the curves provides an estimate of polarization (in $\left.\mathrm{C} / \mathrm{m}^{2}\right)$ at any copolymer fraction $x$ in the range between 0 and 0.5:

$$
\begin{aligned}
& P_{3}^{\mathrm{TrFE}}(x)=0.185-0.20 x+0.069 x^{2}, \\
& P_{3}^{\mathrm{TeFE}}(x)=0.185-0.34 x+0.201 x^{2} .
\end{aligned}
$$

A number of experimental observations, representing the polarization extrapolations to perfect crystallinity in $\beta$-PVDF, $\mathrm{P}(\mathrm{VDF}-\mathrm{TrFE})$, and $\mathrm{P}(\mathrm{VDF}-\mathrm{TeFE})$, are shown in Fig. 10 for comparison. This extrapolation is justified under the assumption that the dipoles in the noncrystalline part are randomly oriented, with zero net polarization. Point (a) refers to the polarization in the $\beta$-PVDF film of Ref. 15 linearly extrapolated from $60 \%$ crystallinity. The extrapolated value of polarization in the film $\left(0.167 \mathrm{C} / \mathrm{m}^{2}\right)$ is approximately $10 \%$ lower than the one obtained in our calculations. Point (b) refers to the $100 \%$ polycrystalline $75-25 \%$ P(VDFTrFE) film of Refs. 20 and 21. No correction was made for the variation of polarization direction among the crystalline grains. Point (c) refers to the polarization of $30-85 \%$ crystalline samples of $73-27 \% \mathrm{P}(\mathrm{VDF}-\mathrm{TrFE})$ extrapolated to $100 \%$ crystallinity by Tajitsu et al. ${ }^{18}$ who estimated the

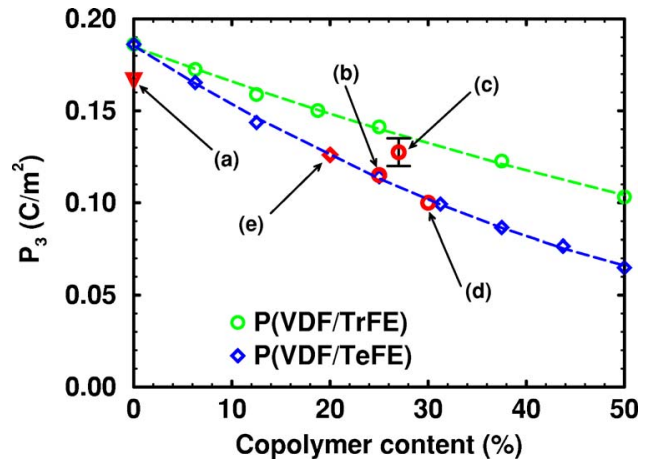

FIG. 10. (Color online) Changes in total polarization in the crystalline $\mathrm{P}(\mathrm{VDF}-\mathrm{TrFE})$ and $\mathrm{P}(\mathrm{VDF}-\mathrm{TeFE})$ models with increasing copolymer concentration. The dashed lines represent the fitted curves of Eqs. (6). Extrapolations of experimental polarizations to $100 \%$ crystallinity are shown for comparison: (a) $\beta$-PVDF film of Ref. 15, (b) polycrystalline $75-25 \%$ P(VDF-TrFE) film of Refs. 20 and 21, (c) $73-27 \% \mathrm{P}$ (VDF-TrFE) sample of Ref. 18, (d) 70-30\% P(VDFTrFE) film of Ref. 41, and (e) 80-20\% P(VDF-TeFE) sample of Ref. 19. See text for discussion.

polarization of a fully crystalline sample to be $0.120-0.135 \mathrm{C} / \mathrm{m}^{2}$. Point (d) refers to a highly crystalline 70-30\% P(VDF-TrFE) Langmuir-Blodget (LB) ultrathin film of Ref. 41. No extrapolation was made here because the crystallinity of the sample is not known. Of these three points, (c) exhibits the best agreement with the calculations. Equation (6a) estimates the polarization in $73-27 \% \mathrm{P}(\mathrm{VDF}-\mathrm{TrFE})$ to be $0.136 \mathrm{C} / \mathrm{m}^{2}$, which is almost within the error bar of the experimental extrapolation. Comparing with the points (b) and (d), the computed polarizations are higher by $22 \%$ and $30 \%$, respectively. The latter large discrepancy is most probably due to the fact that the 68-nm-thin LB film has not yet reached bulklike polarization. Finally, point (e) refers to the polarization obtained in 43-80\% crystalline $80-20 \%$ P(VDFTeFE) samples that were extrapolated to perfect crystallinity in Ref. 19. This point shows an excellent agreement with the calculated polarization. In general, considering that our models represent ideal crystalline systems with no defects and ferroelectric domains, Fig. 10 demonstrates that our computations give an accurate upper-bound estimate of polarization in highly crystalline $\mathrm{P}(\mathrm{VDF}-\mathrm{TrFE})$ and $\mathrm{P}(\mathrm{VDF}-\mathrm{TeFE})$ structures through a wide range of copolymer concentrations, and that they can be used as a guide to grow $\beta$-PVDF-type crystalline materials with predetermined polar properties.

\section{CONCLUSIONS}

We used the Wannier function formalism of the modern theory of polarization to carry out a comprehensive investigation of polar properties of $\beta$-PVDF-type chains and crystalline structures. It was explicitly demonstrated that the polarization strengths of VDF and copolymer monomers dramatically depend on their environment. This collective polarization enhancement varies from almost zero in a chain to $50 \%$ in a crystal. The latter fact explains why many earlier 
models of $\beta$-PVDF, which were fitted to a sum of bonddipole moments of an isolated VDF unit, underestimate the polar properties of bulk polymers. We also studied the effects of weakly polar TrFE and nonpolar TeFE monomers in $\beta$-PVDF crystals, mapping out the evolution of the P(VDFcopolymer) structural and polar properties through a wide range of experimentally useful copolymer concentrations. Analogously to $\beta$-PVDF, these structures exhibit strong collective polarization enhancement, which has a weakly parabolic dependence on the copolymer concentration.

\section{ACKNOWLEDGMENTS}

S.M.N. is grateful to Stephen Ducharme, Karin Rabe, David Vanderbilt, and Morrel Cohen for many useful discussions and valuable suggestions. This work was supported in part by the Office of Naval Research and the Mathematical, Information and Computational Sciences Division, Office of Advanced Scientific Computing Research of the U.S. Department of Energy under Contract No. DE-AC0500OR22725 with UT-Battelle. The calculations were carried out at ARSC and ERDC DoD Supercomputing Centers.
${ }^{1}$ For a few recent results see, e.g., K. J. Choi et al., Science 306, 1005 (2004); H. N. Lee, H. M. Christen, M. F. Chisholm, C. M. Rouleau, and D. H. Lowndes, Nature (London) 433, 395 (2005).

${ }^{2}$ A. J. Lovinger, Science 220, 1115 (1983).

${ }^{3}$ T. Furukawa, IEEE Trans. Electr. Insul. 24, 375 (1989).

${ }^{4}$ R. G. Kepler and R. A. Anderson, Adv. Phys. 41, 1 (1992).

${ }^{5}$ Ferroelectric Polymers, edited by H. S. Nalwa (Dekker, New York, 1995).

${ }^{6}$ G. Eberle, H. Schmidt, and W. Eisenmenger, IEEE Trans. Dielectr. Electr. Insul. 3, 624 (1996).

${ }^{7}$ G. A. Samara, Solid State Phys. 56, 239 (2001).

${ }^{8}$ H. Kakutani, J. Polym. Sci., Part A-2 8, 1177 (1970).

${ }^{9}$ F. Mopsik and M. G. Broadhurst, J. Appl. Phys. 46, 4204 (1975).

${ }^{10}$ K. Tashiro, M. Kobayashi, H. Tadokoro, and E. Fukada, Macromolecules 13, 691 (1980).

${ }^{11}$ C. K. Purvis and P. L. Taylor, Phys. Rev. B 26, 4547 (1982); J. Appl. Phys. 54, 1021 (1983).

${ }^{12}$ R. Al-Jishi and P. L. Taylor, J. Appl. Phys. 57, 897 (1985); 57, 902 (1985).

${ }^{13}$ J. D. Carbeck, D. J. Lacks, and G. C. Rutledge, J. Chem. Phys. 103, 10347 (1995).

${ }^{14}$ J. D. Carbeck and G. C. Rutledge, Polymer 37, 5089 (1995).

${ }^{15}$ To the best of our knowledge, the most recent synthesis techniques produce films with crystallinity of about $60 \%$. See K. Nakamura, D. Sawai, Y. Watanabe, D. Taguchi, Y. Takahashi, T. Furukawa, and T. Kanamoto, J. Polym. Sci., Part B: Polym. Phys. 41, 1701 (2003).

${ }^{16}$ S. M. Nakhmanson, M. B. Nardelli, and J. Bernholc, Phys. Rev. Lett. 92, 115504 (2004).

${ }^{17}$ N. Karasawa and W. A. Goddard III, Macromolecules 25, 7268 (1992).

${ }^{18}$ Y. Tajitsu, H. Ogura, A. Chiba, and T. Furukawa, Jpn. J. Appl. Phys., Part 1 26, 554 (1987).

${ }^{19}$ S. Tasaka and S. Miyata, J. Appl. Phys. 57, 906 (1985).

${ }^{20}$ H. Ohigashi, K. Omote, and T. Gomyo, Appl. Phys. Lett. 66, 3281 (1995).

${ }^{21}$ K. Omote, H. Ohigashi, and K. Koga, J. Appl. Phys. 81, 2760 (1997).

${ }^{22}$ A. V. Bune, V. M. Fridkin, S. Ducharme, L. M. Blinov, S. P. Palto, A. V. Sorokin, S. G. Yudin, and A. Zlatkin, Nature (London) 391, 874 (1998).

${ }^{23}$ See, for example, Q. M. Zhang, V. Bharti, and X. Zhao, Science 280, 2101 (1998); R. Casalini and C. M. Roland, J. Polym. Sci.,
Part B: Polym. Phys. 40, 1975 (2002).

${ }^{24}$ R. D. King-Smith and D. Vanderbilt, Phys. Rev. B 47, R1651 (1993); R. Resta, Rev. Mod. Phys. 66, 899 (1994).

${ }^{25}$ It is possible to use multiple Berry-phase calculations or employ the density functional perturbation theory approach to compute ionic Born effective charges for the system, which allow for various types of analysis of polarization contributions. However, in addition to substantial computational costs, such results are often only approximate, e.g., because one has to average over different atomic planes to obtain polarization in perovskite layers. For polymeric systems this method can be more exact, but Wannier functions provide a more transparent and computationally cheaper alternative.

${ }^{26}$ In both cases (using Berry phases or WF analysis) the electronic polarization in the direction $\alpha$ is obtained modulo $2 e R_{\alpha} / V$, due to arbitrariness in the choice of the phases of the Bloch functions $\psi_{i k}$. A physically meaningful value is obtained by taking a polarization difference between two states of the system.

${ }^{27}$ N. Marzari and D. Vanderbilt, Phys. Rev. B 56, 12847 (1997).

${ }^{28}$ I. Souza, N. Marzari, and D. Vanderbilt, Phys. Rev. B 65, 035109 (2002).

${ }^{29}$ P. L. Silvestrelli, Phys. Rev. B 59, 9703 (1999).

${ }^{30}$ F. Gygi, J.-L. Fattebert, and E. Schwegler, Comput. Phys. Commun. 155, 1 (2003).

${ }^{31}$ E. L. Briggs, D. J. Sullivan, and J. Bernholc, Phys. Rev. B 54, 14362 (1996).

${ }^{32}$ J. P. Perdew, K. Burke, and M. Ernzerhof, Phys. Rev. Lett. 77, 3865 (1996).

${ }^{33}$ N. Troullier and J. L. Martins, Phys. Rev. B 43, 1993 (1991).

${ }^{34}$ L. Kleinman and D. M. Bylander, Phys. Rev. Lett. 48, 1425 (1982).

${ }^{35}$ M. Fuchs and M. Scheffler, Comput. Phys. Commun. 119, 67 (1998).

${ }^{36}$ PWSCF is available from http://www.pwscf.org

${ }^{37}$ D. Vanderbilt, Phys. Rev. B 41, R7892 (1990).

${ }^{38}$ According to our tests, the calculated polarization is insensitive to small errors in the lattice constants, at least in the PVDF-type systems.

${ }^{39}$ H. M. G. Correia and M. M. D. Ramos, Comput. Mater. Sci. 33, 224 (2005).

${ }^{40}$ R. Hasegawa, Y. Takahashi, Y. Chatani, and H. Tadokoro, Polym. J. (Singapore) 3, 600 (1972).

${ }^{41}$ H. Kliem and R. Tadros-Morgane, J. Phys. D 38, 1860 (2005). 